\title{
Bilateral absence of fallopian tube segments: an uncommon condition
}

\section{Tejashri Shrotri*, Meenakshi Ahuja}

Department of Obstetrics and Gynecology, Apollo Cradle Royale Nehru Enclave, New Delhi, Delhi, India

Received: 10 May 2018

Accepted: 03 June 2018

\section{*Correspondence:}

Dr. Tejashri Shrotri,

E-mail: drtejashrisb@gmail.com

Copyright: (c) the author(s), publisher and licensee Medip Academy. This is an open-access article distributed under the terms of the Creative Commons Attribution Non-Commercial License, which permits unrestricted non-commercial use, distribution, and reproduction in any medium, provided the original work is properly cited.

\begin{abstract}
Congenital abnormalities of fallopian tube are rare among the abnormalities of female urogenital system. Bilateral absence of fallopian tube segments is an extremely uncommon condition. The authors report one such rare case diagnosed during diagnostic laparoscopy during evaluation of infertility. On one side, it was completely absent; while on the other side, it was partially absent simulating a formal tubal ligation. To the best of authors knowledge, this is an extremely unusual condition with very few reports available in literature.
\end{abstract}

Keywords: Adnexal torsion, Congenital anomaly, Fallopian tube absence, Infertility

\section{INTRODUCTION}

Congenital abnormalities of female reproductive tract most commonly manifest as some type of uterine anomalies. ${ }^{1}$ Structural abnormalities of fallopian tube are, however, very rare. Partial or complete absence of fallopian tube is usually asymptomatic and are typically diagnosed on hysterosalpingography or during diagnostic laparoscopy during evaluation of infertility. ${ }^{1}$ Authors report an extremely rare case of bilateral absence of the fallopian tube segments.

\section{CASE REPORT}

A 37-years old female presented to us with history of inability to conceive despite unprotected regular intercourse for one and half years. She had no history of abdomino-pelvic surgery or acute pelvic pain and her menstrual cycles were regular within 28 to 32 days intervals. There was no history of dysmenorrhea, dyspareunia and no history suggestive of pelvic inflammatory disease or endometriosis. On pelvic examination, the external genitalia, vagina and cervix was normal. The uterus was normal sized, mobile.
Transvaginal ultrasound revealed a normal uterus and both ovaries appeared normal.

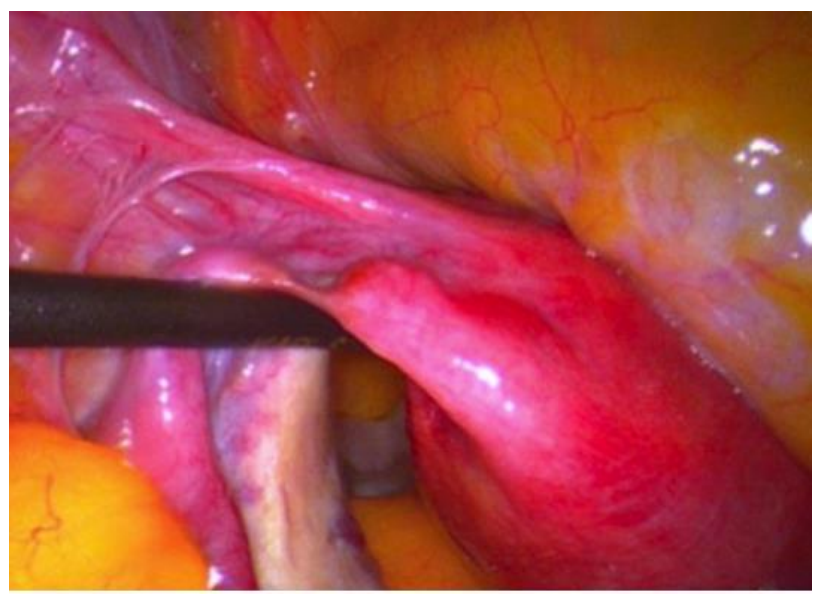

The right fallopian tube had an intact $1 \mathrm{~cm}$ proximal portion, but the ampullary portion was absent simulating a formal tubal ligation.

Figure 1: Diagnostic laparoscopic view of right fallopian tube. 
Basic infertility workup of the couple was normal except sono-salpingography which demonstrated that bilaterally the fluid could not go beyond the cornua. It was decided to perform diagnostic hystero-laparoscopy. Hysteroscopy demonstrated that the cavity was normal. Endometrium appeared normal. Left ostium was seen but right one could not be visualized. On diagnostic laparoscopy, there was absence of ampullary portion of the right fallopian tube simulating a formal tubal ligation (Figure 1).

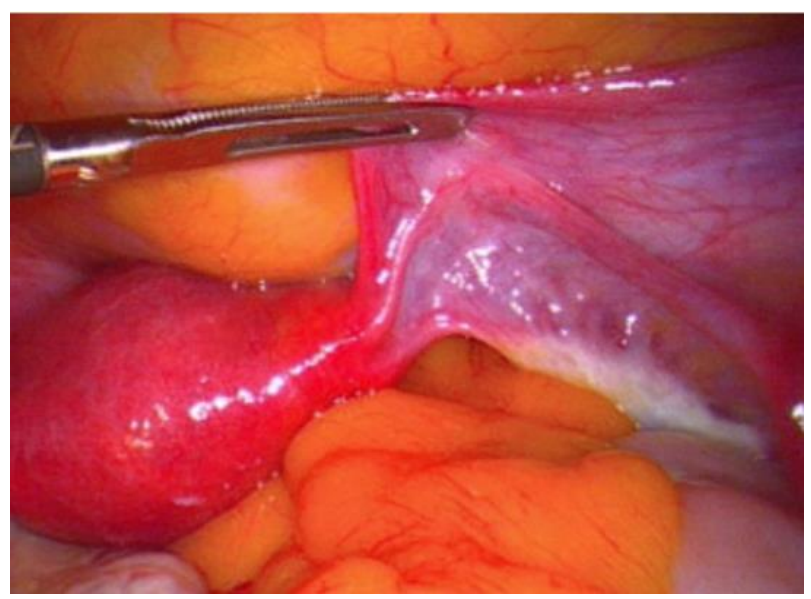

Figure 2: Diagnostic laparoscopic view showing an unicornuate uterus with absent left fallopian tube.

On the left side, the tube was absent (Figure 2). The uterus was unicornate with rudimentary horn on right side. The ultrasound abdomen did not reveal any renal anomaly. The patient was referred for in vitro fertilization and she conceived after two cycles. She had an uneventful antenatal period and delivered a healthy child by caesarean section.

\section{DISCUSSION}

The prevalence of congenital Mullerian duct anomalies ranges from 0.001 to $10 \%$ in the general population and from $8-10 \%$ in women with an adverse reproductive history. ${ }^{2}$ Among the congenital abnormalities of female genital tract, structural abnormalities of fallopian tube are, however, very rare. ${ }^{1}$ Congenital tubal abnormalities can be divided into three groups:

- Complete absence

- Partial or segmental absence

- Duplication, accessory ostia and tubes, multiple Lumina and diverticula.

Complete absence of one fallopian tube is frequently associated with uterine anomalies (e.g. bicornuate uterus). It has been found associated with absence of the ipsilateral ovaries. ${ }^{3,4}$ Autoamputation due to torsion is postulated to be responsible. Partial or segmental absence is very uncommon. ${ }^{1}$ Regarding etiopathogenesis of this uncommon entity, two possible causes have been proposed: congenital absence or torsion. ${ }^{1}$ Developmental abnormalities have been observed in the female reproductive tract, including the fallopian tubes, ovaries, uterus, cervix, vagina and external genitalia. Usually, abnormalities include organs that originate from the Mullerian/paramesonephric ducts. In the sixth week of gestation, the bilateral Mullerian ducts migrate towards the midline, meet, form luminal structures, fuse and finally form the uterus and upper one-fifth of the vagina.

Rostrally, the Mullerian ducts form fallopian tubes. Any disturbance in the migration, fusion or resorption of these ducts may result in a Mullerian anomaly. ${ }^{5}$ Congenital absence is frequently associated with other developmental anomalies of the mesonephric and paramesonephric ducts.

Paramesonephric duct defects result in failure of development of varying portions of fallopian tube. Inadequate blood supply to the caudal part of paramesonephric duct during embryonic development may result if failure of fallopian tube formation. ${ }^{1}$ Combined congenital absence of mesonephric and paramesonephric ducts results in the absence of the uterine horn, fallopian tube, kidney and ureter on the affected side. ${ }^{1}$

Another postulated pathophysiological mechanism is torsion of the uterine adnexal structures. ${ }^{3,4}$ Torsion of the fallopian tube and/or ovary may occur in childhood or in utero (ref). Asymptomatic torsion may lead to avascular necrosis and resorption of the fallopian tubes., ${ }^{3,4}$. The suggested causes for torsion of fallopian tubes include intrinsic factors, like excessive length or spiral course of fallopian tubes or long mesosalpinx and extrinsic causes such as par ovarian cyst, pelvic congestion, etc. However, bilateral isolated torsion of fallopian tubes is extremely rare. Present patient did not give any history suggestive of torsion.

For most cases reported in literature till date, the etiology for this entity remains unclear. However, in present case, in the presence of uterine anomaly; a developmental etiology seems more plausible than a torsion or a vascular accident. Though unilateral absence of fallopian tube with normal contralateral tube have less influence on fertility; the bilateral absence of fallopian tubes is usually discovered during workup for infertility. It is usually diagnosed on diagnostic laparoscopy, as in present case.

\section{CONCLUSION}

Bilateral absence of fallopian tube segments is an extremely rare condition, usually diagnosed during workup for infertility. In the presence of associated uterine anomalies, developmental malformation seems to be the most likely etiology.

Funding: No funding sources

Conflict of interest: None declared

Ethical approval: Not required 


\section{REFERENCES}

1. Yazawa H, Yabe M, Endo S, Hayashi S. A case of congenital unilateral partial absence of fallopian tube. Fukushima J Med Sci. 2010;56(1):44-9.

2. Ribeiro SC, Tormena RA, Peterson TV, Gonzáles MD, Serrano PG, Almeida JA, et al. Müllerian duct anomalies: review of current management. Sao Paulo Med J. 2009;127(2):92-6.

3. Paternoster DM, Costantini W, Uglietti A, Vasile C, Bocconi L. Congenital or torsion-induced absence of Fallopian tubes. Two case reports. Minerva Ginecol. 1998;50(5):191-4.
4. Eustace DL. Congenital absence of fallopian tube and ovary. Europ J Obstet Gynecol Reprod Biol. 1992;46(2-3):157-9.

5. Simpson JL. Genetics of the female reproductive ducts. Am J Med Genetics. 1999;89(4):224-39.

Cite this article as: Shrotri T, Ahuja M. Bilateral absence of fallopian tube segments: an uncommon condition. Int J Reprod Contracept Obstet Gynecol 2019;8:335-7. 